\title{
Prevention and Treatment of Acute Kidney Injury in Patients Undergoing Cardiac Surgery: A Systematic Review
}

\author{
Meyeon Park ${ }^{a, b}$ Steven G. Coca ${ }^{a, b}$ Sagar U. Nigwekar ${ }^{c}$ Amit X. Garg ${ }^{d-f}$ \\ Susan Garwood ${ }^{\mathrm{g}}$ Chirag R. Parikha, b \\ ${ }^{a}$ Clinical Epidemiology Research Center, Veterans Affairs Medical Center, West Haven, Conn., \\ ${ }^{b}$ Department of Medicine, Yale University School of Medicine, New Haven, Conn., and \\ 'Department of Medicine, University of Rochester School of Medicine, Rochester, N.Y., USA; \\ ${ }^{\mathrm{d} D i v i s i o n}$ of Nephrology and ${ }^{\mathrm{e}}$ Department of Epidemiology and Biostatistics, University of Western Ontario, \\ London, Ont., and ${ }^{\mathrm{f} I n s t i t u t e}$ for Clinical Evaluative Sciences, Toronto, Ont., Canada; \\ ${ }^{9}$ Department of Anesthesiology, Yale University School of Medicine, New Haven, Conn., USA
}

\section{Key Words}

Acute kidney injury, prevention · Cardiac surgery ·

Healthcare costs

\begin{abstract}
Background: Acute kidney injury (AKI) is common in patients undergoing cardiac surgery and is associated with a high rate of death, long-term sequelae and healthcare costs. We conducted a systematic review of randomized controlled trials for strategies to prevent or treat AKI in cardiac surgery. Methods: We screened Medline, Scopus, Cochrane Renal Library, and Google Scholar for randomized controlled trails in cardiac surgery for prevention or treatment of AKI in adults. Results: We identified 70 studies that contained a total of 5,554 participants published until November 2008. Most studies were small in sample size, were single-center, focused on preventive strategies, and displayed wide variation in AKI definitions. Only $26 \%$ were assessed to be of high quality according to the Jadad criteria. The types of strategies with possible protective efficacy were dopaminergic agents, vasodilators, anti-inflammatory agents, and pump/perfusion strategies. When analyzed separately, dopamine and $\mathrm{N}$ acetylcysteine did not reduce the risk for AKI. Conclusions:
\end{abstract}

This summary of all the literature on prevention and treatment strategies for AKI in cardiac surgery highlights the need for better information. The results advocate large, good-quality, multicenter studies to determine whether promising interventions reliably reduce rates of acute renal replacement therapy and mortality in the cardiac surgery setting.

Copyright $\odot 2010$ S. Karger AG, Basel

\section{Introduction}

Acute kidney injury (AKI) is a frequent and important complication in hospitalized patients, occurring in up to $5 \%$ of all patients [1]. The incidence of AKI is especially high in patients undergoing cardiac surgery, reaching $50 \%$ by some definitions [2]. The mortality rate in this population is $1-5 \%$ in patients who develop AKI and up to $24 \%$ in patients who require acute renal replacement therapy for AKI [3]. In addition, the mortality rate in cardiac surgery patients with renal injury increases progressively with the degree of renal impairment [4], and AKI is an independent predictor of mortality after cardiac surgery [5]. AKI doubles the total postoperative cost of

\section{KARGER}

(C) 2010 S. Karger AG, Basel

Fax +4161306 1234 E-Mail karger@karger.ch www.karger.com www.karger.com/ajn
Chirag Parikh, MD, PhD

Section of Nephrology, Yale University and VAMC

950 Campbell Ave, Mail Code 151B, Bldg 35 A, Room 219

West Haven, CT 06516 (USA)

Tel. +1 203932 5711, ext. 4300, Fax +1 203937 4932, E-Mail Chirag.parikh@yale.edu 
cardiac surgery patients and nearly doubles intensive care unit costs [2]. Thus, for many reasons any reduction in risk of AKI would be beneficial, but methods to prevent AKI in cardiac surgery patients have not been established.

There are several reasons to conduct clinical trials and study AKI after cardiac surgery. The timing of injury is known; the injury is homogenous in nature relative to other populations in which AKI is frequently studied; and about 800,000 patients undergo cardiac surgery worldwide each year, allowing for large sample sizes and providing a unique opportunity for controlled interventions [6]. The predominant causes of AKI are hypoperfusion and inflammation due to cardiopulmonary bypass (CPB). CPB has also been shown to cause AKI due to nonpulsatile flow causing vasoconstriction and ischemic renal injury [7]. However, even patients undergoing surgery off CPB ('off-pump') are at risk for AKI, suggesting alternative mechanisms for injury.

Given the large population of cardiac surgery patients and the substantial impact of AKI in this population, efforts to treat AKI through various interventions have been attempted. However, no single agent has been shown to prevent AKI in cardiac surgery. Previous systematic reviews have examined AKI in cardiac surgery, but these have focused on individual interventions only $[8,9]$. Other reviews have examined AKI in the broader perioperative period of cardiothoracic and abdominal surgery, which use disparate surgical techniques and may introduce more heterogeneity into the study sample [10]. We conducted this review to evaluate the conduct and outcomes of clinical trials of AKI prevention and treatment in cardiac surgery, to highlight the strengths and limitations of the current evidence, and to guide an agenda for future research.

\section{Methods}

We conducted, analyzed, and reported this systematic review in accordance with consensus guidelines [11].

\section{Data Sources}

We screened Medline (1950 to November 2008), Scopus (19662008), Cochrane Renal Library, and Google Scholar for the relevant studies. Reference lists and bibliographical data from all retrieved articles and reviews were also searched. The terms 'kidney diseases', 'cardiovascular surgical procedures', 'cardiopulmonary bypass', and 'renoprotection' were used. The search strategy in Scopus used the terms 'renal protection', 'renoprotec', 'acute kidney failure', 'kidney failure', 'kidney diseases', 'kidney disease', 'cardiovascular surgery', 'cardiovascular procedures', and 'car- diopulmonary bypass'. An expert librarian was consulted for assistance in conducting a comprehensive search to identify randomized control trials investigating preventive and therapeutic measures for AKI in cardiac surgery. Two reviewers (M.P. and S.N.) independently screened the citations and those considered potentially relevant were retrieved for full-text review.

Study Eligibility and Selection

Articles published as full manuscripts in English were included. The studies were limited to humans and to all adults from age 19 to 80 years and above, with no upper age limit specified. Randomized controlled trials (RCTs) involving patients undergoing cardiac surgery (coronary artery bypass grafting, CABG; valve surgery, or combined CABG/valve surgery; elective, emergent, or not specified) were included. Studies that assessed kidney injury by methods of serum creatinine or creatinine clearance/glomerular filtration rate were eligible. Eligible interventions included methods of prevention or treatment of AKI administered anytime before, during, or after surgery. These included medical therapies as well as procedure-based therapies such as CPB modification and early renal replacement therapy. Healthcare service interventions such as level of care preceding or following surgery were not eligible.

Comparison was with no therapy, placebo, or with standard care for the institution, such as maximal hydration. Review outcomes were incidence of AKI (defined by individual study authors using one of several definitions for AKI) or change in serum creatinine, creatinine clearance, or GFR, incidence of acute renal replacement therapy and mortality. Renal outcomes were abstracted regardless of whether they were a primary or secondary trial outcome.

Patients with all degrees of renal function prior to surgery were included. Studies describing outcomes for patients who were on renal replacement therapy prior to surgery or who had received a kidney transplant were excluded from analysis.

\section{Data Extraction}

We used a comprehensive data collection form to record study characteristics: type of surgery (CABG, valve surgery, combined CABG/valve, elective, urgent), demographics of the participants (age, sex), and baseline mean serum creatinine or GFR. We characterized the timing of the intervention as preoperative (commencing outside operating room), intraoperative (commencing after anesthesia induction, initiated within 30 min before or after $\mathrm{CPB}$ ), or postoperative (commencing after surgery, outside the operating room). Interventions were described by principal agent, route, and dose administered and were grouped according to their principal mechanism of action as follows: interventions that increase renal blood flow (vasodilators); interventions that induce natriuresis or diuresis; anti-inflammatory interventions, and interventions that work through other mechanisms of actions. Outcomes for creatinine, creatinine clearance/GFR, and incidence of acute renal replacement therapy and mortality were recorded for each study. Data extraction was performed independently by two reviewers (M.P. and S.N.) and disagreements were resolved by consensus.

Quality Assessment

All RCTs were evaluated for study quality using the Jadad score [12]. This score awards one point each for randomization, 
appropriateness of randomization, blinding, appropriateness of blinding, and description of withdrawal and dropouts, with a maximum score of 5. As studies involving pump strategies do not uniformly describe blinding techniques, we confirmed the quality characteristics of these studies using randomization, allocation concealment, blinded outcome assessment, and intention-totreat analysis [8]. Patients excluded and lost to follow-up were recorded. Using these criteria, we classified the studies as good, moderate, and low quality (table 1).

\section{Data Analysis}

All trials were two- or three-arm interventions and administered parallel in design. Three-arm trials involved two separate interventions analyzed against a single control group. Outcomes were reviewed separately for prevention and treatment cohorts. Overall results for each intervention class were mathematically pooled using techniques that accounted for within- and betweenstudy heterogeneity $[13,14]$. For studies that reported a continuous outcome (e.g. change in creatinine, creatinine clearance, or eGFR), we compared the standard difference in means in treatment and control groups. For trials that only reported continuous outcomes, we converted the standard difference in means to log odds ratios via the following formula: $\log$ odds ratio $=\pi \times$ standard difference/square root [3]. The variance calculation was based on the following: $\log$ odds $\mathrm{SE}=$ square $\operatorname{root}(\pi \wedge 2 \times$ standard difference $\mathrm{SE} \wedge 2 / 3$ ). The log odds variance was equal to the $\log$ odds $\mathrm{SE} \wedge 2$. This allowed pooling of studies that only reported continuous outcomes with those that reported categorical outcomes. We formally assessed heterogeneity of treatment effects between studies with the Cochrane Q and the $\mathrm{I}^{2}$ statistics. Publication bias was not assessed due to high statistical heterogeneity $[15,16]$. All analyses were performed using Comprehensive Meta Analysis Software Version 2.0 (Englewood, N.J., USA).

\section{Results}

\section{Retrieval of Studies and Study Characteristics}

Our search of Medline yielded a total of 169 citations for individual review. Scopus retrieved 194 citations for review. Additional searches of the Cochrane Renal Library (Issue 4, 2008), PubMed, and Google Scholar produced 29 additional citations for review. We also studied reference lists and bibliographical data from all retrieved articles and reviews for any additional relevant material (fig. 1). A total of 70 studies (5,554 patients) met eligibility criteria [17-86] (table 1).

Twenty-one different countries were represented, with the highest number of studies coming from the UK $(\mathrm{n}=$ 9), Turkey $(n=9)$, Germany $(n=9)$, USA $(n=7)$, and Italy $(\mathrm{n}=7)$. Sixty-four studies involved CABG, and 27 involved valvular surgeries. Study size ranged widely from 14 to 388 patients. Nine studies comprised groups of 20 patients or fewer; 17 studies had more than 100 patients, and only 6 studies had more than 200 patients. The ma- jority (93\%), including the larger studies, were single-center studies and only 5 trials included more than one center (table 1, footnote). Five studies described an industry sponsor. Sixty-six studies examined prevention strategies and 4 examined treatment strategies. Patients with preoperative chronic kidney disease (CKD) were excluded from 10 studies. Definitions of AKI were not uniform. Criteria for initiation of acute renal replacement therapy were not standardized across studies.

Thirty studies were designed to analyze the effects of interventions that primarily increase renal blood flow (vasodilators). These interventions included dopamine, dopexamine, fenoldopam, angiotensin-converting enzyme inhibitors (captopril, enalaprilat), diltiazem, prostacyclin, nifedipine, PGE-1, sodium nitroprusside and theophylline. All of the studies amongst the vasodilator cohort were designed to evaluate effects on prevention of AKI and none of the studies in this cohort evaluated effects on treatment of an established AKI.

Twelve studies were designed to analyze the effects of interventions that primarily induce natriuresis or diuresis or both. These interventions included atrial natriuretic peptide, brain natriuretic peptide, urodilatin, and diuretic agents (loop diuretics and mannitol). Ten studies amongst this natriuretic cohort were designed to evaluated effects on prevention of AKI and 3 studies were designed to evaluate effects on treatment of an established AKI.

Fourteen studies were designed to analyze the effects of interventions that primarily counteract inflammation (anti-inflammatory agents). These interventions included $\mathrm{N}$-acetylcysteine, aspirin, glutathione, corticosteroids, and leukodepletion. All of the studies amongst this antiinflammatory cohort were designed to evaluate effects on prevention of AKI.

Interventions that have been studied previously but could not be assigned to one of the above 3 cohorts included clonidine, albumin infusion, isotonic saline infusion, insulin therapy, early continuous veno-venous hemofiltration and interventions such as the off-pump technique and pulsatile technique. Amongst these only 1 study addressing the role of continuous veno-venous hemofiltration was designed to treat an established AKI and all other interventions were studied in a prevention setting.

\section{Quality}

There were 18 studies of good quality, 15 studies of moderate quality, and 37 studies of low quality. Fortyseven studies (67\%) had $5 \%$ or fewer patients excluded or 
Table 1. Characteristics of included randomized controlled trials

\begin{tabular}{|c|c|c|c|c|c|c|c|}
\hline Source & Intervention & Trial type & $\begin{array}{l}\text { Inclusion } \\
\text { of CKD } \\
\text { patients }\end{array}$ & $\begin{array}{l}\text { Number } \\
\text { of } \\
\text { patients }\end{array}$ & $\begin{array}{l}\text { Mean } \\
\text { age } \\
\text { years }\end{array}$ & $\begin{array}{l}\text { Male } \\
\%\end{array}$ & $\begin{array}{l}\text { Jadad } \\
\text { score }^{1}\end{array}$ \\
\hline \multicolumn{8}{|l|}{ Anti-inflammatory } \\
\hline Adabag et al. [18], 2008 & NAC & Prevention & Yes & 102 & 71 & 100 & 5 \\
\hline Amano et al. [19], 1994 & Glutathione & Prevention & No & 19 & 57.5 & NR & 3 \\
\hline Barr and Kolodner [22], 2008 & NAC, fenoldopam & Prevention & Yes & 79 & 74.2 & 65.8 & 4 \\
\hline Bolcal et al. [25], 2006 & Leukodepletion & Prevention & Yes & 50 & 56.9 & 72 & 3 \\
\hline Burns et al. [28], 2005 & NAC & Prevention & Yes & 295 & 69.1 & 78.7 & 5 \\
\hline Fischer et al. [41], 2005 & NAC & Prevention & NR & 40 & 66 & 77.5 & 5 \\
\hline Gerrah et al. [44], 2004 & Aspirin & Prevention & Yes & 94 & 68.5 & 78 & 1 \\
\hline Haase et al. [45], 2007 & NAC & Prevention & Yes & 60 & 68.6 & 73.3 & 5 \\
\hline Loef et al. [54], 2004 & Dexamethasone & Prevention & No & 20 & 63.7 & 85 & 3 \\
\hline McBride et al. [58], 2004 & Methylprednisolone & Prevention & Yes & 36 & 61.45 & 97.2 & 2 \\
\hline Ristikankare et al. [67], 2006 & $\mathrm{NAC}$ & Prevention & Yes & 77 & 70.5 & 80.5 & 5 \\
\hline Sisillo et al. [73], 2008 & NAC & Prevention & Yes & 254 & 72.5 & 49 & 5 \\
\hline Tang et al. [78], 2002 & Leukodepletion & Prevention & Yes & 44 & 63.5 & 85 & 3 \\
\hline Wijeysundera et al. [81], 2007 & NAC & Prevention & Yes & 177 & 73.5 & 59.5 & 5 \\
\hline \multicolumn{8}{|l|}{ Natriuretics/diuretics } \\
\hline Chen et al. [33], 2007 & Nesiritide & Prevention & Yes & 36 & 77.5 & 61.5 & 4 \\
\hline Hayashida et al. [47], 2000 & ANP & Prevention & NR & 18 & 60.3 & 80 & 2 \\
\hline Mahesh et al. [55], 2008 & Furosemide & Prevention & Yes & 42 & 71.3 & 73.8 & 4 \\
\hline Mentzer et al. [59], 2007 & Nesiritide & Prevention & Yes & 272 & 63.9 & 78.5 & 5 \\
\hline Meyer et al. [60], 1997 & Urodilatin & Treatment & Yes & 14 & 59.4 & NR & 3 \\
\hline Nuutinen and Hollmen [64], 1976 & Furosemide & Prevention & NR & 45 & 35.1 & 53.3 & 1 \\
\hline Sezai et al. [70], 2000 & ANP & Prevention & NR & 40 & 63.5 & 87.5 & 3 \\
\hline Sezai et al. [71], 2007 & ANP & Prevention & No & 124 & 67.25 & 70.2 & 3 \\
\hline Sirivella et al. [72], 2000 & Mannitol, furosemide, DA & Treatment & Yes & 100 & 71 & 62.5 & 3 \\
\hline Smith et al. [74], 2008 & Mannitol & Prevention & Yes & 47 & 74.7 & 72.3 & 5 \\
\hline Sward et al. [77], 2004 & ANP & Treatment & Yes & 59 & 69.7 & 71 & 5 \\
\hline Yallop et al. [84], 2008 & Mannitol & Prevention & No & 40 & 63.2 & 75 & 5 \\
\hline \multicolumn{8}{|l|}{ Vasodilators } \\
\hline Abe et al. [17], 1993 & PGE1 & Prevention & NR & 20 & 55 & NR & 3 \\
\hline Amano et al. [20], 1995 & Diltiazem & Prevention & NR & 23 & 54.4 & NR & 2 \\
\hline Berendes et al. [23], 1997 & Dopexamine & Prevention & NR & 44 & 61.5 & 59.1 & 3 \\
\hline Bergman et al. [24], 2002 & Diltiazem & Prevention & Yes & 24 & 72.5 & 92 & 5 \\
\hline Bove et al. [27], 2005 & DA, fenoldopam & Prevention & Yes & 80 & 68.5 & 72.5 & 4 \\
\hline Caimmi et al. [29], 2003 & Fenoldopam & Prevention & Yes & 160 & 69 & 66.3 & 2 \\
\hline Carcoana et al. [30], 2003 & DA, mannitol & Prevention & Yes & 100 & 64 & 72 & 5 \\
\hline Cogliati et al. [34], 2007 & Fenoldopam & Prevention & Yes & 193 & 70 & 0.6 & 5 \\
\hline Colson et al. [35], 1990 & ACE inhibitor & Prevention & Yes & 18 & 58 & 100 & 3 \\
\hline Costa et al. [36], 1990 & DA, nitroprusside & Prevention & Yes & 36 & 58.6 & NR & 3 \\
\hline Dehne et al. [37], 2001 & Dopexamine & Prevention & NR & 36 & 63.4 & 100 & 2 \\
\hline Dural et al. [39], 2000 & DA, mannitol & Prevention & No & 36 & 53.7 & 63.9 & 3 \\
\hline Gatot et al. [43],2004 & $\mathrm{DA}$ & Prevention & Yes & 82 & 65 & NR & 5 \\
\hline Halpenny et al. [46], 2001 & Fenoldopam & Prevention & No & 31 & 64 & 24.3 & 3 \\
\hline Kaya et al. [48], 2007 & Sodium nitroprusside & Prevention & Yes & 240 & 61.1 & 63.7 & 5 \\
\hline Kramer et al. [50], 2002 & Theophylline & Prevention & No & 56 & 60.4 & 75 & 4 \\
\hline Lassnigg et al. [52], 2000 & DA, furosemide & Prevention & Yes & 123 & 63.3 & 68.3 & 4 \\
\hline Lema et al. [53], 1998 & DA, phenylephrine & Prevention & Yes & 17 & 65 & 85 & 2 \\
\hline Monaco et al. [61], 2005 & $\mathrm{DA}$ & Prevention & Yes & 67 & 65.8 & 70.7 & 2 \\
\hline Morgera et al. [62], 2002 & Prostacyclin & Prevention & NR & 34 & 61.5 & 91.2 & 3 \\
\hline Myles et al. [63], 1993 & $\mathrm{DA}$ & Prevention & Yes & 52 & 61.6 & 63 & 4 \\
\hline Piper et al. [66], 2003 & DA, diltiazem & Prevention & Yes & 60 & 67.7 & 66.7 & 5 \\
\hline Ryckwaert et al. [68], 2001 & ACE inhibitor & Prevention & Yes & 14 & 63.2 & 92.9 & 4 \\
\hline
\end{tabular}


Table 1 (continued)

\begin{tabular}{|c|c|c|c|c|c|c|c|}
\hline Source & Intervention & Trial type & $\begin{array}{l}\text { Inclusion } \\
\text { of CKD } \\
\text { patients }\end{array}$ & $\begin{array}{l}\text { Number } \\
\text { of } \\
\text { patients }\end{array}$ & $\begin{array}{l}\text { Mean } \\
\text { age } \\
\text { years }\end{array}$ & $\begin{array}{l}\text { Male } \\
\%\end{array}$ & $\begin{array}{l}\text { Jadad } \\
\text { score }^{1}\end{array}$ \\
\hline Sumeray et al. [76], 2001 & DA & Prevention & Yes & 36 & 63.4 & 91.7 & 5 \\
\hline Tang et al. [80], 1999 & $\mathrm{DA}$ & Prevention & No & 40 & 58.7 & 60 & 2 \\
\hline Witczak et al. [82], 2008 & Nifedipine & Prevention & Yes & 20 & 66.8 & 80 & 5 \\
\hline Woo et al. [83], 2002 & DA & Prevention & Yes & 42 & 65.5 & 58.5 & 3 \\
\hline Yavuz et al. [85], 2002 & DA & Prevention & No & 22 & 56.1 & 91 & 2 \\
\hline Yavuz et al. [86], 2002 & DA, diltiazem & Prevention & No & 60 & 59.3 & 86.7 & 2 \\
\hline \multicolumn{8}{|l|}{ Operative } \\
\hline Ascione et al. [21], 1999 & Off-pump & Prevention & Yes & 50 & 61.6 & 90 & 2 \\
\hline Carrier et al. [31], 2003 & Off-pump & Prevention & Yes & 65 & 70 & 76.9 & 3 \\
\hline Celik et al. [32], 2005 & Off-pump & Prevention & Yes & 60 & 67.1 & 51.7 & 3 \\
\hline Kocakulak et al. [49], 2005 & Off-pump pulsatile & Prevention & NR & 40 & 53.7 & 77.5 & 2 \\
\hline Masoumi et al. [57], 2008 & Off-pump & Prevention & NR & 124 & 58.9 & 83 & 3 \\
\hline Onorati et al. [65], 2007 & Off-pump pulsatile & Prevention & Yes & 100 & 68 & 93 & 3 \\
\hline Sajja et al. [69], 2007 & Off-pump & Prevention & Yes & 116 & 60.3 & 88.8 & 3 \\
\hline Straka et al. [75], 2004 & Off-pump & Prevention & Yes & 388 & 62.5 & 81.5 & 3 \\
\hline Tang et al. [79], 2002 & Off-pump & Prevention & Yes & 40 & 66 & 80 & 3 \\
\hline \multicolumn{8}{|l|}{ Other } \\
\hline Boldt et al. [26], 2008 & Albumin & Prevention & Yes & 50 & 82.5 & 50 & 2 \\
\hline Demirkilic et al. [38], 2004 & Early CVVHDF & Treatment & Yes & 61 & 60.5 & NR & 2 \\
\hline Durmaz et al. [40], 2003 & RRT & Prevention & Yes & 44 & 56.2 & 79.4 & 2 \\
\hline Gandhi et al. [42], 2007 & Insulin & Prevention & NR & 371 & 63 & 69 & 5 \\
\hline Kulka et al. [51], 1996 & Clonidine & Prevention & NR & 50 & 57.5 & 78 & 3 \\
\hline Marathias et al. [56], 2006 & Hydration with $0.5 \%$ normal saline & Prevention & Yes & & 64.1 & 95 & 2 \\
\hline
\end{tabular}

Multicenter trials: Burns et al. [28], Kaya et al. [48], Mentzer et al. [59], Meyer et al. [60], and Sward et al. [77]. Industry sponsor: Barr and Kolodner [22], Chen et al. [33], Gandhi et al. [42], Halpenny et al. [46], and Kramer et al. [50].

$\mathrm{NAC}=\mathrm{N}$-Acetylcysteine; ANP = atrial natriuretic peptide; $\mathrm{DA}=$ dopamine; $\mathrm{ACE}=$ angiotensin-converting enzyme; $\mathrm{NR}=$ not reported; $\mathrm{RRT}=$ renal replacement therapy; $\mathrm{CVVHDF}=$ continuous veno-venous hemodiafiltration.

${ }^{1}$ Jadad score awards one point for randomization, appropriateness of randomization, blinding, appropriateness of blinding, and description of withdrawal and dropouts, with a maximum score of 5 . lost to follow-up, with 34 (49\%) of these studies having $0 \%$ excluded. Fifteen studies (21\%) had $>5 \%$ excluded or lost to follow-up, with a range of 6-29\%. Seven studies did not report patients excluded or lost to follow-up. All studies assessed kidney function uniformly between the intervention and control groups. The frequency of assessing kidney function was variable between studies, ranging from a frequency of every $8 \mathrm{~h}$ (as in a good-quality study [77]) to measurements on days 1, 5, and 15 [69], with 9 studies not reporting the method of assessment.

\section{AKI}

The incidence of AKI as a dichotomous outcome was reported in 22 studies (2,674 patients). AKI was not uniformly defined in these studies. Continuous endpoints were reported in 43 studies (2,148 patients). Amongst the prevention cohort trials, there were 9 interventions that commenced preoperatively, 53 administered intraoperatively, and 5 postoperatively (table 2 ).

The following agents from the vasodilator group were associated with a reduction in AKI: fenoldopam and angiotensin-converting enzyme inhibitors; whereas the other vasodilator agents were noted to have no effect on the incidence of AKI. Anti-inflammatory agents including $\mathrm{N}$-acetylcysteine were not associated with any reduction in AKI. The following agents from the natriuretic and/or diuretic cohort were associated with a reduction in AKI: atrial natriuretic peptide, B-natriuretic peptide, urodilatin; whereas the remaining agents from this group were noted to have no effect on the incidence of AKI. 
Fig. 1. Flowchart of study selection.

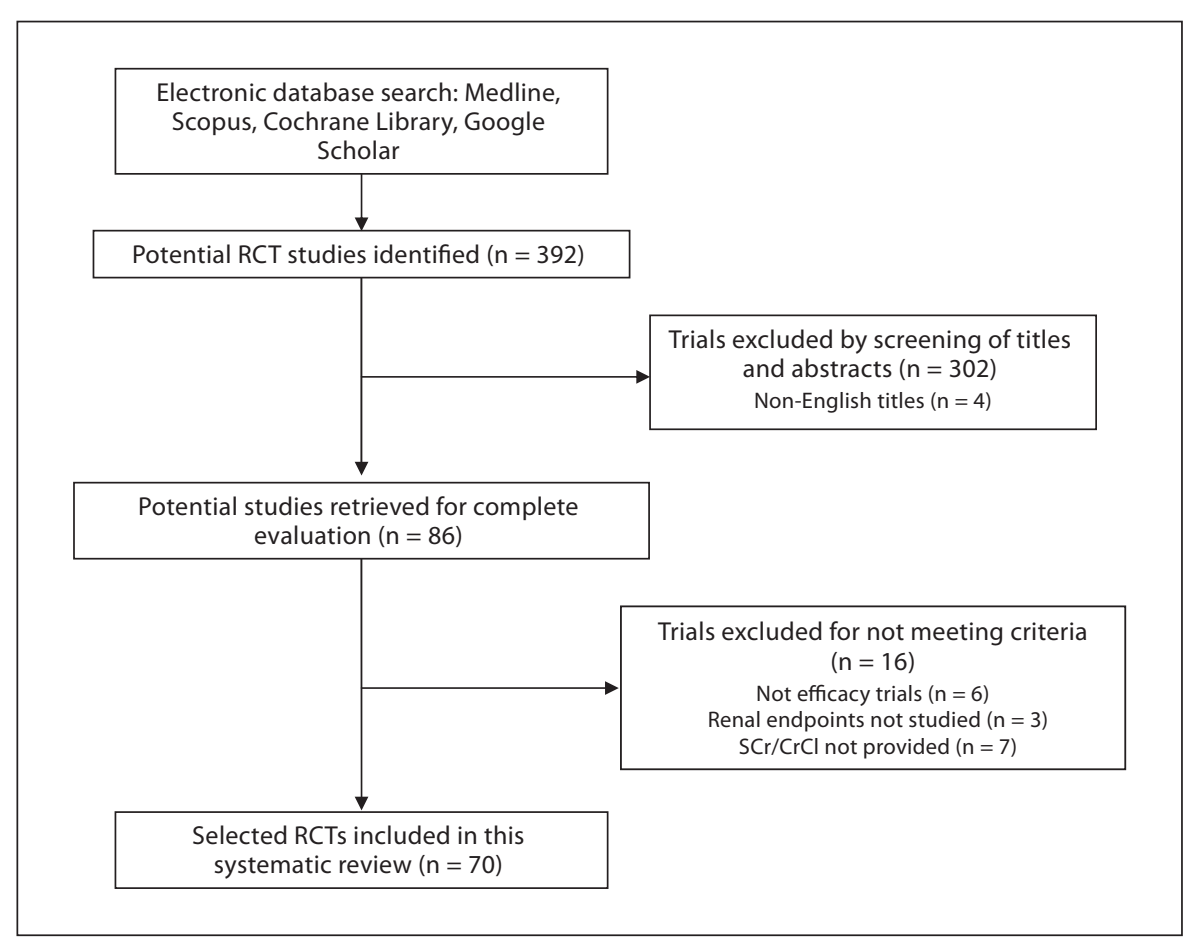

Amongst the other interventions that were reviewed, the off-pump surgical technique and pulsatile flow techniques were associated with a reduction in the incidence of AKI, whereas interventions such as clonidine, albumin infusion, isotonic saline infusion, and insulin therapy were not associated with a reduction in AKI.

\section{Acute Renal Replacement Therapy}

The incidence of acute renal replacement therapy was provided in 21 studies comprising 2,172 patients $[18,25$, 27, 28, 32-34, 40, 45, 52, 55, 56, 60, 61, 67, 69, 71, 75, 77, 81, 82]. Predefined criteria for acute renal replacement therapy initiation were provided in 7 of these studies and were not uniform across studies [25, 27, 40, 55, 56, 77, 81]. In 1 case, initiation was determined by blinded nephrologists without specific parameters described [81]. In another study, prophylactic hemodialysis was the intervention and thus acute renal replacement therapy parameters were needed for both groups [40]. Other studies did not describe criteria for initiation of acute renal replacement therapy.

None of the individual trials showed a clear benefit in terms of reducing the incidence of acute renal replacement therapy in the cardiac surgery setting and none of the trials were adequately powered to study this outcome.

Cardiac Surgery-Associated Acute Kidney Injury

\section{Mortality}

Data on mortality were reported in 18 studies comprising 2,227 patients. As mortality is a competing endpoint for AKI, we considered any study that described both of these outcomes as a composite endpoint; however, no such studies were found.

None of the cohorts (vasodilator agents, anti-inflammatory agents, natriuretic/diuretic agents and agents with other mechanisms of action) demonstrated a reduction in mortality and none of the trials were adequately powered to study this outcome.

\section{Comment}

This systematic review demonstrates that a large number of RCTs to prevent or treat AKI after cardiac surgery have been performed over the past 30 years. The majority of trials were small, single-center, methodologically and statistically heterogeneous, rated to be of low methodological quality, and most were not powered to detect differences in hard endpoints such as mortality and acute renal replacement therapy. In addition, the definitions of AKI were quite variable and many trials instead examined continuous changes in kidney function. However, analysis of these existing trials shows that there may be

Am J Nephrol 2010;31:408-418 
Table 2. Study details by intervention

\begin{tabular}{|c|c|c|c|c|c|}
\hline Source & Intervention & Control & $\begin{array}{l}\text { Timing } \\
\text { of inter- } \\
\text { vention }\end{array}$ & $\begin{array}{l}\text { Baseline renal } \\
\text { function }^{1}\end{array}$ & Outcomes \\
\hline \multicolumn{6}{|l|}{ Anti-inflammatory } \\
\hline Adabag et al. [18], 2008 & NAC p.o., $600 \mathrm{mg}$ b.i.d. $\times 14$ doses & Placebo & Pre & $1.9 \pm 0.7 / 40 \pm 10$ & AKI, D \\
\hline Amano et al. [19], 1994 & Glutathione i.v., $200 \mathrm{mg} / \mathrm{kg}$ & Placebo & I & $1.1 \pm 0.2 / \mathrm{NR}$ & Mean SCr \\
\hline Barr and Kolodner [22] & Fenoldopam i.v., $0.1 \mu \mathrm{g} / \mathrm{kg} / \mathrm{min}$ & Placebo + NAC or & I & $\mathrm{NR} / 34 \pm 2$ & Mean $\mathrm{CrCl}$ \\
\hline 2008 & & Fenoldopam + NAC & & & \\
\hline Bolcal et al. [25], 2006 & Leukodepletion & $\mathrm{CPB}$ alone & $\mathrm{I}$ & $1.8 \pm 0.6 / \mathrm{NR}$ & Mean SCr, R \\
\hline Burns et al. [28], 2005 & NAC i.v., $600 \mathrm{mg} \times 4$ doses & $5 \%$ dextrose & I & $1.2 \pm 0.4 / \mathrm{NR}$ & AKI, R, D \\
\hline Fischer et al. [41], 2005 & NAC i.v., $100 \mathrm{mg} / \mathrm{kg}, 20 \mathrm{mg} / \mathrm{kg} / \mathrm{h}$ infusion & Placebo & I & $1.0 \pm 0.4 / \mathrm{NR}$ & Mean SCr \\
\hline Gerrah et al. [44], 2004 & Aspirin p.o., $100 \mathrm{mg}$ daily until surgery & Placebo & Pre & $2.8 \pm 1.6 / 31 \pm 14$ & Mean S r \\
\hline Haase et al. [45], 2007 & NAC i.v., $300 \mathrm{mg} / \mathrm{kg}$ over $24 \mathrm{~h}$ & $5 \%$ dextrose & I & $1.0 \pm 0.3 / 78 \pm 24$ & AKI, R \\
\hline Loef et al. [54], 2004 & Dexamethasone i.v., $1 \mathrm{mg} / \mathrm{kg}$ plus $0.5 \mathrm{mg} / \mathrm{kg}$ & Placebo & $\mathrm{I}$ & $1.0 \pm 0.2 / 104 \pm 10$ & Mean $\mathrm{CrCl}$ \\
\hline McBride et al. [58], 2004 & Methylprednisolone i.v., $30 \mathrm{mg} / \mathrm{kg}$ & Placebo & $\mathrm{I}$ & $1.1 \pm 0.2$ & Mean SCr \\
\hline Ristikankare et al. [67] & NAC i.v., $150 \mathrm{mg} / \mathrm{kg}, 50 \mathrm{mg} / \mathrm{kg}$ & Placebo & I & $1.5 \pm 0.4 / \mathrm{NR}$ & AKI, R, D \\
\hline 2006 & $100 \mathrm{mg} / \mathrm{kg}+$ & & & & \\
\hline Sisillo et al. [73], 2008 & NAC i.v., $1,200 \mathrm{mg}$ every $12 \mathrm{~h} \times 4$ boluses & Placebo & I & $1.3 \pm 0.4 / 46 \pm 8$ & AKI, D \\
\hline Tang et al. [78], 2002 & Leukodepletion & CPB alone & I & $1.1 \pm 0.2 / \mathrm{NR}$ & Mean SCr \\
\hline $\begin{array}{l}\text { Wijeysundera et al. [81] } \\
2007\end{array}$ & $\begin{array}{l}\text { NAC i.v., } 100 \mathrm{mg} / \mathrm{kg} \text { bolus, } 20 \mathrm{mg} / \mathrm{kg} / \mathrm{h} \\
\text { infusion }\end{array}$ & $5 \%$ dextrose & I & $1.4 \pm 0.4 / 44 \pm 11$ & AKI, R \\
\hline
\end{tabular}

\section{Diuretics}

Mahesh et al. [55], 2008

Nuutinen and Hollmen [64], 1976

Sirivella et al. [72], 2000

Smith et al. [74], 2008

Yallop et al. [84], 2008
Furosemide i.v., $4 \mathrm{mg} / \mathrm{h}$

Furosemide i.v., varying doses w/UOP $<40 \mathrm{ml} / \mathrm{h}$

Furosemide, ethacrynic acid, bumetanide

Mannitol i.v., $0.5 \mathrm{~g} / \mathrm{kg}$

Mannitol i.v., $5 \mathrm{ml} / \mathrm{kg}, 10 \%$ solution
Normal saline

Placebo

Osmitrol, furosemide, DA

Hartmann's solution

Hartmann's solution
Vasodilators

Abe et al. [17], 1993

Amano et al. [20], 1995

Berendes et al. [23], 1997

Bergman et al. [24], 2002

Bove et al. [27], 2005

Caimmi et al. [29], 2003

Carcoana et al. [30], 2003

Chen et al. [33], 2007

Cogliati et al. [34], 2007

Colson et al. [35], 1990

Costa et al. [36], 1990

Dehne et al. [37], 2001

Dural et al. [39], 2000

Gatot et al. [43], 2004

Halpenny et al. [46], 2001

Hayashida et al. [47], 2000

Kaya et al. [48], 2007

Kramer et al. [50], 2002

Lassnigg et al. [52], 2000

Lema et al. [53], 1998

Mentzer et al. [59], 2007

Meyer et al. [60], 1997

Monaco et al. [61], 2005

Morgera et al. [62], 2002

Myles et al. [63], 1993
PGE-1 i.v., $0.02 \mu \mathrm{g} / \mathrm{kg}-\mathrm{min}$

Diltiazem i.v., $0.1 \mathrm{mg} / \mathrm{kg}$ bolus,

$2 \mathrm{mcg} / \mathrm{kg} / \mathrm{min}$ inf.

Dopexamine i.v., 0.5, 1.0, $2.0 \mu \mathrm{g} / \mathrm{kg} / \mathrm{min}$

Diltiazem i.v., $0.25 \mathrm{mg} / \mathrm{kg}$ bolus,

$1.7 \mu \mathrm{g} / \mathrm{kg} / \mathrm{min}$ infusion

Fenoldopam i.v., $0.05 \mu \mathrm{g} / \mathrm{kg} / \mathrm{min}$

Fenoldopam i.v., $0.1-0.3 \mu \mathrm{g} / \mathrm{kg} / \mathrm{min}$

$\mathrm{DA} \pm$ mannitol i.v., $2 \mu \mathrm{g} / \mathrm{kg} / \mathrm{min}$ or $1 \mathrm{~g} / \mathrm{kg}$

Nesiritide i.v., $0.005 \mu \mathrm{g} / \mathrm{kg} / \mathrm{min}$

Fenoldopam i.v., $0.1 \mu \mathrm{g} / \mathrm{kg} / \mathrm{min}$

Captopril p.o., $100 \mathrm{mg}$ b.i.d. $\times 2$ days

DA i.v., $2.5 \mu \mathrm{g} / \mathrm{kg} / \mathrm{min}$

Dopexamine i.v., $1 \mu \mathrm{g} / \mathrm{kg} / \mathrm{min}$

DA i.v., $0.3 \mu \mathrm{g} / \mathrm{kg} / \mathrm{min}$

DA i.v., 3-5 $\mu \mathrm{g} / \mathrm{kg} / \mathrm{min}$

Fenoldopam i.v., $0.1 \mu \mathrm{g} / \mathrm{kg} / \mathrm{min}$

ANP i.v., $0.05 \mu \mathrm{g} / \mathrm{kg} / \mathrm{min}$

SNP i.v., $0.1 \mathrm{mg} / \mathrm{kg} / \mathrm{h}$

Theophylline i.v., $0.25 \mathrm{mg} / \mathrm{kg} / \mathrm{h}$

DA i.v., $2 \mu \mathrm{g} / \mathrm{kg} / \mathrm{min}+$

DA i.v., $2 \mu \mathrm{g} / \mathrm{kg} / \mathrm{min}$

Nesiritide i.v., $0.01 \mu \mathrm{g} / \mathrm{kg} / \mathrm{min}$

Urodilatin i.v., $20 \mathrm{ng} / \mathrm{kg} / \mathrm{min} \times 7$ days

DA i.v., $3 \mu \mathrm{g} / \mathrm{kg} / \mathrm{min}$

Prostacyclin i.v., $2 \mathrm{ng} / \mathrm{kg} / \mathrm{min}$

DA i.v., $200 \mu \mathrm{g} / \mathrm{min}$
Normal saline

Placebo

Placebo

Placebo

DA i.v., $2.5 \mu \mathrm{g} / \mathrm{kg} / \mathrm{min}$

DA or dobutamine i.v.,

renal doses+

Placebo

Placebo

Placebo

Placebo

Placebo; DA; nitroprusside

Placebo

No treatment;

$20 \%$ mannitol i.v., $1 \mathrm{mg} / \mathrm{kg} / \mathrm{h}$

Normal saline

Placebo

Control

Normal saline

Normal saline;

furosemide $0.5 \mu \mathrm{g} / \mathrm{kg} / \mathrm{min}$

Phenylephrine

Placebo

Placebo

Placebo

Placebo

$5 \%$ dextrose
Normal saline
$1.1 \pm 0.3 / 65 \pm 34$

$\mathrm{NR} / 89 \pm 17$

AKI, R, D

Mean $\mathrm{CrCl}$

\begin{tabular}{|c|c|c|}
\hline I & $1.1 \pm 0.3 / 65 \pm 34$ & AKI, R, D \\
\hline I & $\mathrm{NR} / 89 \pm 17$ & Mean $\mathrm{CrCl}$ \\
\hline Post & $1.8 \mathrm{NR} / \mathrm{NR}$ & $\mathrm{R}$ \\
\hline I & $1.8 \pm 0.3 / 33 \pm 10$ & Mean SCr \\
\hline I & $1.1 \pm 0.2 / \mathrm{NR}$ & Mean SCr \\
\hline I & $\mathrm{NR} / 89 \pm 8$ & Mean $\mathrm{CrCl}$ \\
\hline I & NR/90 \pm 10 & Mean $\mathrm{CrCl}$ \\
\hline Pre & NR/NR & Mean $\mathrm{CrCl}$ \\
\hline I & $1.8 \pm 0.1 / \mathrm{NR}$ & Mean SCr \\
\hline I & $1.6 \pm 0.7 / 50 \pm 21$ & AKI, R, D \\
\hline I & $1.8 \pm 0.3 / 51 \pm 22$ & Mean SCr \\
\hline I & $1.1 \pm 0.2 / 96 \pm 27$ & Mean SCr \\
\hline I & $1.7 \pm 0.6 / 40 \pm 11$ & AKI, R, D \\
\hline I & $1.8 \pm 0.4 / 39 \pm 10$ & AKI, R \\
\hline Pre & $1.2 \pm 0.2 / 107 \pm 12$ & Mean $\mathrm{CrCl}$ \\
\hline I & $N R / 37 \pm 10$ & Mean $\mathrm{CrCl}$ \\
\hline I & $\mathrm{NR} / 61 \pm 13$ & Mean $\mathrm{CrCl}$ \\
\hline I & $1.1 \pm 0.4 / \mathrm{NR}$ & Mean SCr \\
\hline Post & $1.1 \pm 0.2 / \mathrm{NR}$ & Mean SCr \\
\hline Post & $1.1 \pm 0.2 / 107 \pm 36$ & Mean $\mathrm{CrCl}$ \\
\hline I & $\mathrm{NR} / 48 \pm 7$ & Mean $\mathrm{CrCl}$ \\
\hline I & $1 \pm 0.2 / 77 \pm 21$ & AKI \\
\hline I & $0.8 \mathrm{NR} / \mathrm{NR}$ & AKI \\
\hline I & $1.0 \pm 0.2 / 96 \pm 37$ & AKI, R, D \\
\hline I & $1.5 \pm 0.4 / 74 \pm 37$ & Mean SCr \\
\hline I & $1.1 \pm 0.4 / 80 \pm 29$ & Mean SCr, D \\
\hline Post & $2.7 \pm 0.6$ & Mean SCr, R, D \\
\hline Pre & $1.9 \pm 0.2$ & Mean SCr, R, D \\
\hline I & NR/96 \pm 22 & $\mathrm{AKI}$ \\
\hline I & $1.0 \pm 0.2 / \mathrm{NR}$ & Mean SCr \\
\hline
\end{tabular}


Table 2 (continued)

\begin{tabular}{|c|c|c|c|c|c|}
\hline Source & Intervention & Control & $\begin{array}{l}\text { Timing } \\
\text { of inter- } \\
\text { vention }\end{array}$ & $\begin{array}{l}\text { Baseline renal } \\
\text { function }^{1}\end{array}$ & Outcomes \\
\hline Piper et al. [66], 2003 & DA i.v., $2.5 \mu \mathrm{g} / \mathrm{kg} / \mathrm{min}$ & $\begin{array}{l}\text { Normal saline; diltiazem i.v., } \\
2 \mu \mathrm{g} / \mathrm{kg} / \mathrm{min}\end{array}$ & Post & $\mathrm{NR} / 74 \pm 34$ & Mean $\mathrm{CrCl}$ \\
\hline Ryckwaert et al. [68], 2001 & Enalaprilat i.v., $1 \mathrm{mg}$ every $6 \mathrm{~h}$ over 2 days & Placebo & $\mathrm{I}$ & $1.2 \pm 0.2 / 70 \pm 14$ & Mean $\mathrm{CrCl}$ \\
\hline Sezai et al. [70], 2000 & Human ANP i.v., $0.03-0.05 \mu \mathrm{g} / \mathrm{kg} / \mathrm{min}$ & Placebo & $\mathrm{I}$ & $\mathrm{NR} / 91 \pm 20$ & AKI \\
\hline Sezai et al. [71], 2007 & Human ANP i.v., 0.02 g/dl/min & NR & $\mathrm{I}$ & NR/NR & $\mathrm{R}, \mathrm{D}$ \\
\hline Sumeray et al. [76], 2001 & DA i.v., $2.5 \mu \mathrm{g} / \mathrm{kg} / \mathrm{min}$ & $5 \%$ dextrose & I & $\mathrm{NR} / 77 \pm 3$ & Mean $\mathrm{CrCl}$ \\
\hline Sward et al. [77], 2004 & Recombinant hANP i.v., 50 ng/kg/min & $\mathrm{NR}$ & $\begin{array}{l}\text { treat- } \\
\text { ment }\end{array}$ & $1.2 \pm 0 / \mathrm{NR}$ & $\mathrm{R}, \mathrm{D}$ \\
\hline Tang et al. [80], 1999 & DA i.v., $2.5-4.0 \mu \mathrm{g} / \mathrm{kg} / \mathrm{min}$ & Placebo & I & $1.3 \pm 0.1 / \mathrm{NR}$ & Mean SCr \\
\hline Witczak et al. [82], 2008 & Nifedipine i.v., $0.25-0.60 \mu \mathrm{g} / \mathrm{kg} / \mathrm{min}$ & Placebo & $\mathrm{I}$ & $2.7 \pm 1.0 / 35 \pm 10$ & Mean $\mathrm{CrCl}, \mathrm{R}$ \\
\hline Woo et al. [83], 2002 & DA i.v., $3 \mu \mathrm{g} / \mathrm{kg} / \mathrm{min} \times 48 \mathrm{~h}$ & NR & $\mathrm{I}$ & $1.1 \pm 0.2 / \mathrm{NR}$ & Mean SCr \\
\hline Yavuz et al. [85], 2002 & DA i.v., $2 \mu \mathrm{g} / \mathrm{kg} / \mathrm{min}$ & Placebo & Pre & $\mathrm{NR} / 71 \pm 35$ & Mean $\mathrm{CrCl}$ \\
\hline Yavuz et al. [86], 2002 & DA i.v., $200 \mu \mathrm{g} / \mathrm{kg} / \mathrm{min}$ & $\begin{array}{l}\text { Placebo/diltiazem; } \\
\text { diltiazem and DA }\end{array}$ & I & $\mathrm{NR} / 77 \pm 49$ & Mean $\mathrm{CrCl}$ \\
\hline \multicolumn{6}{|l|}{ Operative } \\
\hline Ascione et al. [21], 1999 & Off-pump & On-pump & $\mathrm{I}$ & $\mathrm{NR} / 91 \pm 26$ & Mean $\mathrm{CrCl}$ \\
\hline Carrier et al. [31], 2003 & Off-pump & On-pump & I & NR/NR & AKI, D \\
\hline Celik et al. [32], 2005 & Off-pump & On-pump & $\mathrm{I}$ & $1.4 \pm 0.3 / \mathrm{NR}$ & Mean SCr, R \\
\hline Kocakulak et al. [49], 2005 & Pulsatile flow & Continuous flow & I & $1.5 \pm 1.1 / \mathrm{NR}$ & Mean SCr \\
\hline Masoumi et al. [57], 2008 & Off-pump & On-pump & $\mathrm{I}$ & NR/NR & AKI, D \\
\hline Onorati et al. [65], 2007 & Pulsatile flow & Standard IABP & $\mathrm{I}$ & $1.2 \pm 0.4 / 80 \pm 25$ & AKI \\
\hline Sajja et al. [69], 2007 & Off-pump & On-pump & $\mathrm{I}$ & $1.5 \pm 0.5 / 52 \pm 9$ & AKI, R \\
\hline Straka et al. [75], 2004 & Off-pump & On-pump & $\mathrm{I}$ & NR/NR & $\mathrm{R}, \mathrm{D}$ \\
\hline Tang et al. [79], 2002 & Off-pump & Pulsatile CPB & I & $1.1 \pm 0.2 / \mathrm{NR}$ & Mean SCr \\
\hline \multicolumn{6}{|l|}{ Other } \\
\hline Boldt et al. [26], 2008 & Albumin 5\% i.v., $500 \mathrm{ml}$ & HES 6\% i.v., $500 \mathrm{ml}$ & I & $1.3 \pm 0.4 / 47 \pm 20$ & Mean SCr \\
\hline Demirkilic et al. [38], 2004 & Early CVVHDF & Standard CVVHDF & Post & $>3 \mathrm{NR} / \mathrm{NR}$ & $\mathrm{D}$ \\
\hline Durmaz et al. [40], 2003 & Postoperative prophylactic hemodialysis & $\begin{array}{l}\text { Standard indications for } \\
\text { renal replacement therapy }\end{array}$ & Post & $3.4 \pm 0.8 / \mathrm{NR}$ & AKI, D \\
\hline Gandhi et al. [42], 2007 & Insulin i.v., continuously & Insulin i.v., intermittently & I & NR/NR & AKI \\
\hline Kulka et al. [51], 1996 & Clonidine i.v., $4 \mu \mathrm{g} / \mathrm{kg}$ & Placebo & Pre & NR/94 \pm 19 & Mean $\mathrm{CrCl}$ \\
\hline Marathias et al. [56], 2006 & $0.5 \%$ normal saline i.v., $1 \mathrm{ml} / \mathrm{kg} / \mathrm{h}$ over $12 \mathrm{~h}$ & Fluid restriction & Pre & $3.3 \pm 0.5 / 26 \pm 2$ & AKI, R \\
\hline
\end{tabular}

$\mathrm{NAC}=\mathrm{N}$-Acetylcysteine ANP $=$ atrial natriuretic peptide DA $=$ dopamine; $\mathrm{NR}=$ not reported; $\mathrm{RRT}=$ renal replacement therapy; CVVHDF = continuous veno-venous hemodiafiltration; AKI = acute kidney injury; $\mathrm{D}=$ death; $\mathrm{R}=$ acute renal replacement therapy; Pre = preoperative; Post = postoperative; $\mathrm{I}=$ intraoperative; $\mathrm{CrCl}=$ creatinine clearance; $\mathrm{SCr}=$ serum creatinine; $\mathrm{CPB}=$ cardiopulmonary bypass.
${ }^{1}$ Serum creatinine $\pm \mathrm{SD} /$ creatinine clearance $\pm \mathrm{SD}$. Serum creatinine in $\mathrm{mg} / \mathrm{dl}$ (converted from $\mu \mathrm{mol} / \mathrm{l}$ ) rounded to the nearest 10 th, creatinine clearance in $\mathrm{ml} / \mathrm{min}$ rounded to nearest 1 . For further details of interventions, refer to original references. benefits associated with some interventions for the prevention of AKI. This review calls for good-quality, largepopulation trials of individual or combination of agents.

In general, strategies to prevent AKI were effective if administered preoperatively and intraoperatively. Strategies for treatment of AKI were far less numerous and thus it is difficult to draw conclusions about their efficacy relative to prophylaxis strategies. Our exploratory analyses revealed that most types of prophylactic strategies were protective for AKI. In particular, fenoldopam, ANP/nesiritide and off-pump CABG demonstrated excellent efficacy for the prevention of AKI. There was no evidence for benefit from preoperative administration of dopamine or $\mathrm{N}$-acetylcysteine.

The primary endpoints in the majority of trials contained in this review were continuous changes in creatinine or creatinine clearance/GFR rather than the most clinically important endpoints of acute renal replacement therapy and mortality. Furthermore, in the 22 studies that utilized categorical outcomes for AKI, the definition of AKI was highly variable. Acute renal replacement therapy and/or mortality were considered primary outcomes in only 5 studies; most studies did not have adequate statistical power for non-primary outcomes. 
There are many challenges to consider when designing and executing future trials for the prevention or treatment of AKI in cardiac surgery. The first challenge relates to patient selection. If one chooses to study an intervention that has potential adverse side effects, only those at the highest risk of AKI should be enrolled. These may include patients undergoing redo cardiac surgeries or those with CKD. Second, since AKI may be multifactorial after CPB, multiple agents acting through different pathways may need to be administered simultaneously or in succession in order to effectively reduce AKI. Third, the selection of the correct endpoint for these trials is vital. Early phase 1 and 2 trials should measure surrogate endpoints such a AKI defined by serum creatinine using RIFLE or AKIN criteria or changes in novel biomarkers of AKI. Larger phase 3 and 4 studies should examine the ability of the interventions to reduce hard endpoints, such as dialysis, death, length of stay, and long-term events such as cardiovascular events, CKD, and long-term death.

Our study has several strengths. We performed a comprehensive search to compile relevant studies, screening over 500 citations. Article identification, eligibility assessment, and data abstraction were performed independently and in duplicate, to minimize potential biases inherent in these tasks. Through these methods, we included a large number of studies for systematic review and meta-analysis, encompassing a wider scope than previous reviews evaluating a similar question.

Our review also has some limitations. Only studies written in English were included, resulting in the exclusion of four studies (one in Chinese, one in Japanese, and two in Italian). Trials were in general small, underpow- ered, and of poor methodological quality. Risk factors for AKI, such as CKD, were not consistently reported in a standardized fashion. Trials included in this review had variable definitions of AKI and none of the included trials reported the recently proposed AKIN criteria to define AKI. Future trials should follow these criteria to consistently define AKI and should also have predefined trial criteria for the initiation of renal replacement therapy [89].

\section{Conclusions}

AKI in cardiac surgery patients is common and is associated with significant morbidity and mortality. A method of preventing this common complication is urgently needed. Most studies were underpowered to demonstrate a beneficial effect on acute renal replacement therapy and mortality. The beneficial effect on AKI alone is enough impetus for a more thorough investigation into prophylaxis and treatment strategies. Large, good-quality, multicenter trials needed to demonstrate benefits of prevention of AKI and reduction in rates of acute renal replacement therapy and mortality in the cardiac surgery setting.

\section{Acknowledgment}

The authors would like to thank Mark Gentry, Clinical Support Librarian and Coordinator, Yale University Cushing/Whitney Medical Library. Dr. Parikh was supported by the NIH grant HL-085757.

\section{References}

1 Chertow GM, Levy EM, Hammermeister $\mathrm{KE}$, Grover F, Daley J: Independent association between acute renal failure and mortality following cardiac surgery. Am J Med 1998;104:343-348.

-2 Dasta JF, Kane-Gill SL, Durtschi AJ, Pathak DS, Kellum JA: Costs and outcomes of acute kidney injury (AKI) following cardiac surgery. Nephrol Dial Transplant 2008;23: 1970-1974.

3 Chertow GM, Christiansen CL, Cleary PD, Munro C, Lazarus JM: Prognostic stratification in critically ill patients with acute renal failure requiring dialysis. Arch Intern Med 1995;155:1505-1511.
4 Zanardo G, Michielon P, Paccagnella A, et al: Acute renal failure in the patient undergoing cardiac operation. Prevalence, mortality rate, and main risk factors. J Thorac Cardiovasc Surg 1994;107:1489-1495.

5 Lassnigg A, Schmidlin D, Mouhieddine M, et al: Minimal changes of serum creatinine predict prognosis in patients after cardiothoracic surgery: a prospective cohort study. J Am Soc Nephrol 2004;15:1597-1605.

6 Nalysnyk L, Fahrbach K, Reynolds MW, Zhao SZ, Ross S: Adverse events in coronary artery bypass graft (CABG) trials: a systematic review and analysis. Heart 2003;89:767772 .

7 Abu-Omar Y, Ratnatunga C: Cardiopulmonary bypass and renal injury. Perfusion 2006;21:209-213.
8 Wijeysundera DN, Beattie WS, Djaiani G, et al: Off-pump coronary artery surgery for reducing mortality and morbidity: meta-analysis of randomized and observational studies. J Am Coll Cardiol 2005;46:872-882.

-9 Landoni G, Biondi-Zoccai GG, Marino G, et al: Fenoldopam reduces the need for renal replacement therapy and in-hospital death in cardiovascular surgery: a meta-analysis. J Cardiothorac Vasc Anesth 2008;22:27-33.

10 Zacharias M, Conlon NP, Herbison GP, Sivalingam P, Walker RJ, Hovhannisyan K: Interventions for protecting renal function in the perioperative period. [update of Cochrane Database Syst Rev 2005;3:CD003590.] 
11 Moher D, Cook DJ, Eastwood S, Olkin I, Rennie D, Stroup DF: Improving the quality of reports of meta-analyses of randomised controlled trials: the QUOROM statement. Quality of Reporting of Meta-analyses. Lancet 1999;354:1896-1900.

12 Jadad AR, Moore RA, Carroll D, et al: Assessing the quality of reports of randomized clinical trials: is blinding necessary? Control Clin Trials 1996;17:1-12.

13 DerSimonian R, Laird N: Meta-analysis in clinical trials. Control Clin Trials 1986;7: 177-188.

14 Higgins JP, Thompson SG, Deeks JJ, Altman DG: Measuring inconsistency in meta-analyses. BMJ 2003;327:557-560.

- 15 Terrin N, Schmid CH, Lau J, Olkin I: Adjusting for publication bias in the presence of heterogeneity. Stat Med 2003;22:2113-2126.

$\checkmark 16$ Lau J, Ioannidis JP, Terrin N, Schmid CH, Olkin I: The case of the misleading funnel plot. BMJ 2006;333:597-600.

17 Abe K, Fujino Y, Sakakibara T: The effect of prostaglandin E1 during cardiopulmonary bypass on renal function after cardiac surgery. Eur J Clin Pharmacol 1993;45:217-220.

18 Adabag AS, Ishani A, Koneswaran S, et al: Utility of $\mathrm{N}$-acetylcysteine to prevent acute kidney injury after cardiac surgery: a randomized controlled trial. Am Heart J 2008; 155:1143-1149.

19 Amano J, Suzuki A, Sunamori M: Salutary effect of reduced glutathione on renal function in coronary artery bypass operation. J Am Coll Surg 1994;179:714-720.

20 Amano J, Suzuki A, Sunamori M, Tofukuji M: Effect of calcium antagonist diltiazem on renal function in open heart surgery. Chest 1995; 107:1260-1265.

-21 Ascione R, Lloyd CT, Underwood MJ, Gomes WJ, Angelini GD: On-pump versus offpump coronary revascularization: evaluation of renal function. Ann Thorac Surg 1999;68:493-498.

-22 Barr LF, Kolodner K: N-acetylcysteine and fenoldopam protect the renal function of patients with chronic renal insufficiency undergoing cardiac surgery. Crit Care Med 2008;36:1427-1435.

23 Berendes E, Mollhoff T, Van Aken H, et al: Effects of dopexamine on creatinine clearance, systemic inflammation, and splanchnic oxygenation in patients undergoing coronary artery bypass grafting. Anesth Analg 1997;84:950-957.

- 24 Bergman AS, Odar-Cederlof I, Westman L, Bjellerup P, Hoglund P, Ohqvist G: Diltiazem infusion for renal protection in cardiac surgical patients with preexisting renal dysfunction. J Cardiothorac Vasc Anesth 2002; 16:294-299.

25 Bolcal C, Akay HT, Bingol H, et al: Leukodepletion improves renal function in patients with renal dysfunction undergoing onpump coronary bypass surgery: a prospective randomized study. Thorac Cardiovasc Surg 2007;55:89-93.
26 Boldt J, Brosch C, Rohm K, Lehmann A, Mengistu A, Suttner S: Is albumin administration in hypoalbuminemic elderly cardiac surgery patients of benefit with regard to inflammation, endothelial activation, and long-term kidney function? Anesth Analg 2008;107:1496-1503.

27 Bove T, Landoni G, Calabro MG, et al: Renoprotective action of fenoldopam in high-risk patients undergoing cardiac surgery: a prospective, double-blind, randomized clinical trial. Circulation 2005;111:3230-3235.

-28 Burns KE, Chu MW, Novick RJ, et al: Perioperative $\mathrm{N}$-acetylcysteine to prevent renal dysfunction in high-risk patients undergoing $C A B G$ surgery: a randomized controlled trial. JAMA 2005;294:342-350.

29 Caimmi PP, Pagani L, Micalizzi E, et al: Fenoldopam for renal protection in patients undergoing cardiopulmonary bypass. J Cardiothorac Vasc Anesth 2003;17:491-494.

30 Carcoana OV, Mathew JP, Davis E, et al: Mannitol and dopamine in patients undergoing cardiopulmonary bypass: a randomized clinical trial. Anesth Analg 2003;97: 1222-1229.

- 31 Carrier M, Perrault LP, Jeanmart H, Martineau R, Cartier R, Page P: Randomized trial comparing off-pump to on-pump coronary artery bypass grafting in high-risk patients. Heart Surg Forum 2003;6:E89-E92.

32 Celik JB, Gormus N, Topal A, Okesli S, Solak $\mathrm{H}$ : Effect of off-pump and on-pump coronary artery bypass grafting on renal function. Ren Fail 2005;27:183-188

33 Chen HH, Sundt TM, Cook DJ, Heublein DM, Burnett JC Jr: Low dose nesiritide and the preservation of renal function in patients with renal dysfunction undergoing cardiopulmonary-bypass surgery: a double-blind placebo-controlled pilot study. Circulation 2007;116:134-138.

34 Cogliati AA, Vellutini R, Nardini A, et al: Fenoldopam infusion for renal protection in high-risk cardiac surgery patients: a randomized clinical study. J Cardiothorac Vasc Anesth 2007;21:847-850.

- 35 Colson P, Ribstein J, Mimran A, Grolleau D, Chaptal PA, Roquefeuil B: Effect of angiotensin converting enzyme inhibition on blood pressure and renal function during open heart surgery. Anesthesiology 1990;72: 23-27.

36 Costa P, Ottino GM, Matani A, et al: Lowdose dopamine during cardiopulmonary bypass in patients with renal dysfunction. J Cardiothorac Anesth 1990;4:469-473.

- 37 Dehne MG, Klein TF, Muhling J, Sablotzki A, Osmer C, Hempelmann G: Impairment of renal function after cardiopulmonary bypass is not influenced by dopexamine. Ren Fail 2001;23:217-230.

38 Demirkilic U, Kuralay E, Yenicesu M, et al: Timing of replacement therapy for acute renal failure after cardiac surgery. J Card Surg 2004;19:17-20.
39 Dural O, Ozkara A, Celebioglu b, Kanbak M, Ciliv G, Aypar U: Comparative study of dopamine and mannitol effects on renal function during cardiopulmonary bypass by using $\mathrm{N}$-acetyl-beta-D-glucosaminidase assay. Turk J Med Sci 2000;30:453-457.

-40 Durmaz I, Yagdi T, Calkavur T, et al: Prophylactic dialysis in patients with renal dysfunction undergoing on-pump coronary artery bypass surgery. Ann Thorac Surg 2003;75: 859-864.

-41 Fischer UM, Weissenberger WK, Warters RD, Geissler HJ, Allen SJ, Mehlhorn U: Impact of cardiopulmonary bypass management on postcardiac surgery renal function. Perfusion 2002;17:401-406.

42 Gandhi GY, Nuttall GA, Abel MD, et al: Intensive intraoperative insulin therapy versus conventional glucose management during cardiac surgery: a randomized trial. Ann Intern Med 2007;146:233-243.

43 Gatot I, Abramov D, Tsodikov V, et al: Should we give prophylactic renal-dose dopamine after coronary artery bypass surgery? J Card Surg 2004;19:128-133.

44 Gerrah R, Ehrlich S, Tshori S, Sahar G: Beneficial effect of aspirin on renal function in patients with renal insufficiency postcardiac surgery. J Cardiovasc Surg 2004;45:545-550.

45 Haase M, Haase-Fielitz A, Bagshaw SM, et al: Phase II, randomized, controlled trial of high-dose $\mathrm{N}$-acetylcysteine in high-risk cardiac surgery patients. Crit Care Med 2007;35: 1324-1331.

46 Halpenny M, Lakshmi S, O’Donnell A, O'Callaghan-Enright S, Shorten GD: Fenoldopam: renal and splanchnic effects in patients undergoing coronary artery bypass grafting. Anaesthesia 2001;56:953-960.

-47 Hayashida N, Chihara S, Kashikie H, et al: Effects of intraoperative administration of atrial natriuretic peptide. Ann Thorac Surg 2000;70:1319-1326.

- 48 Kaya K, Oguz M, Akar AR, et al: The effect of sodium nitroprusside infusion on renal function during reperfusion period in patients undergoing coronary artery bypass grafting: a prospective randomized clinical trial. Eur J Cardiothorac Surg 2007;31:290-297.

49 Kocakulak M, Askin G, Kucukaksu S, Tarcan O, Piskin E: Pulsatile flow improves renal function in high-risk cardiac operations. Blood Purif 2005;23:263-267.

-50 Kramer BK, Preuner J, Ebenburger A, et al: Lack of renoprotective effect of theophylline during aortocoronary bypass surgery. $\mathrm{Ne}$ phrol Dial Transplant 2002;17:910-915.

51 Kulka PJ, Tryba M, Zenz M: Preoperative alpha2-adrenergic receptor agonists prevent the deterioration of renal function after cardiac surgery: results of a randomized, controlled trial. Crit Care Med 1996;24:947-952.

52 Lassnigg A, Donner E, Grubhofer G, Presterl E, Druml W, Hiesmayr M: Lack of renoprotective effects of dopamine and furosemide during cardiac surgery. J Am Soc Nephrol 2000;11:97-104. 
53 Lema G, Urzua J, Jalil R, et al: Renal protection in patients undergoing cardiopulmonary bypass with preoperative abnormal renal function. Anesth Analg 1998;86:3-8.

54 Loef BG, Henning RH, Epema AH, et al: Effect of dexamethasone on perioperative renal function impairment during cardiac surgery with cardiopulmonary bypass. Br J Anaesth 2004;93:793-798.

-55 Mahesh B, Yim B, Robson D, Pillai R, Ratnatunga C, Pigott D: Does furosemide prevent renal dysfunction in high-risk cardiac surgical patients? Results of a double-blinded prospective randomised trial. Eur J Cardiothorac Surg 2008;33:370-376.

-56 Marathias KP, Vassili M, Robola A, et al: Preoperative intravenous hydration confers renoprotection in patients with chronic kidney disease undergoing cardiac surgery. Artif Organs 2006;30:615-621.

57 Masoumi M, Saidi MR, Rostami F, Sepahi H, Roushani D: Off-pump coronary artery bypass grafting in left ventricular dysfunction. Asian Cardiovasc Thorac Ann 2008;16:1620.

58 McBride WT, Allen S, Gormley SM, et al: Methylprednisolone favourably alters plasma and urinary cytokine homeostasis and subclinical renal injury at cardiac surgery. Cytokine 2004;27:81-89.

59 Mentzer RM Jr, Oz MC, Sladen RN, et al: Effects of perioperative nesiritide in patients with left ventricular dysfunction undergoing cardiac surgery: the NAPA Trial. J Am Coll Cardiol 2007;49:716-726.

60 Meyer M, Wiebe K, Wahlers T, et al: Urodilatin (INN:ularitide) as a new drug for the therapy of acute renal failure following cardiac surgery. Clin Exp Pharmacol Physiol 1997;24:374-376.

-61 Monaco M, Di Tommaso L, Mottola M, Stassano P, Iannelli G: Clinical outcome for onpump myocardial revascularization in patients with mild renal dysfunction. Thorac Cardiovasc Surg 2005;53:46-51.

62 Morgera S, Woydt R, Kern H, et al: Low-dose prostacyclin preserves renal function in high-risk patients after coronary bypass surgery. Crit Care Med 2002;30:107-112.

63 Myles PS, Buckland MR, Schenk NJ, et al: Effect of 'renal-dose' dopamine on renal function following cardiac surgery. Anaesth Intensive Care 1993;21:56-61.

64 Nuutinen L, Hollmen A: The effect of prophylactic use of furosemide on renal function during open heart surgery. Ann Chir Gynaecol 1976;65:258-266.
65 Onorati F, Presta P, Fuiano G, et al: A randomized trial of pulsatile perfusion using an intra-aortic balloon pump versus nonpulsatile perfusion on short-term changes in kidney function during cardiopulmonary bypass during myocardial reperfusion. Am J Kidney Dis 2007;50:229-238.

66 Piper SN, Kumle B, Maleck WH, et al: Diltiazem may preserve renal tubular integrity after cardiac surgery. Can J Anaesth 2003;50: 285-292.

67 Ristikankare A, Kuitunen T, Kuitunen A, et al: Lack of renoprotective effect of i.v. N-acetylcysteine in patients with chronic renal failure undergoing cardiac surgery. Br J Anaesth 2006;97:611-616.

68 Ryckwaert F, Colson P, Ribstein J, Boccara G Guillon G: Haemodynamic and renal effects of intravenous enalaprilat during coronary artery bypass graft surgery in patients with ischaemic heart dysfunction. Br J Anaesth 2001;86:169-175.

69 Sajja LR, Mannam G, Chakravarthi RM, et al: Coronary artery bypass grafting with or without cardiopulmonary bypass in patients with preoperative non-dialysis dependent renal insufficiency: a randomized study. J Thorac Cardiovasc Surg 2007;133:378-388.

70 Sezai A, Shiono M, Orime Y, et al: Low-dose continuous infusion of human atrial natriuretic peptide during and after cardiac surgery. Ann Thorac Surg 2000;69:732-738

71 Sezai A, Hata M, Wakui S, et al: Efficacy of continuous low-dose hANP administration in patients undergoing emergent coronary artery bypass grafting for acute coronary syndrome. Circ J 2007;71:1401-1407.

72 Sirivella S, Gielchinsky I, Parsonnet V: Mannitol, furosemide, and dopamine infusion in postoperative renal failure complicating cardiac surgery. Ann Thorac Surg 2000;69:501506.

73 Sisillo E, Ceriani R, Bortone F, et al: N-acetylcysteine for prevention of acute renal failure in patients with chronic renal insufficiency undergoing cardiac surgery: a prospective, randomized, clinical trial. Crit Care Med 2008;36:81-86

74 Smith MN, Best D, Sheppard SV, Smith DC: The effect of mannitol on renal function after cardiopulmonary bypass in patients with established renal dysfunction. Anaesthesia 2008;63:701-704.

75 Straka Z, Widimsky P, Jirasek K, et al: Offpump versus on-pump coronary surgery: final results from a prospective randomized study PRAGUE-4. Ann Thorac Surg 2004; 77:789-793.

76 Sumeray M, Robertson C, Lapsley M, Bomanji J, Norman AG, Woolfson RG: Low dose dopamine infusion reduces renal tubular injury following cardiopulmonary bypass surgery. J Nephrol 2001;14:397-402.
77 Sward K, Valsson F, Odencrants P, Samuelsson $\mathrm{O}$, Ricksten SE: Recombinant human atrial natriuretic peptide in ischemic acute renal failure: a randomized placebo-controlled trial. Crit Care Med 2004;32:13101315.

78 Tang AT, Alexiou C, Hsu J, Sheppard SV, Haw MP, Ohri SK: Leukodepletion reduces renal injury in coronary revascularization: a prospective randomized study. Ann Thorac Surg 2002;74:372-377.

79 Tang AT, Knott J, Nanson J, Hsu J, Haw MP, Ohri SK: A prospective randomized study to evaluate the renoprotective action of beating heart coronary surgery in low risk patients. Eur J Cardiothorac Surg 2002;22:118-123.

80 Tang AT, El-Gamel A, Keevil B, Yonan N, Deiraniya AK: The effect of 'renal-dose' dopamine on renal tubular function following cardiac surgery: assessed by measuring retinol binding protein (RBP). Eur J Cardiothorac Surg 1999;15:717-721.

81 Wijeysundera DN, Beattie WS, Rao V, Granton JT, Chan CT: N-acetylcysteine for preventing acute kidney injury in cardiac surgery patients with pre-existing moderate renal insufficiency. Can J Anaesth 2007;54: $872-881$.

82 Witczak BJ, Hartmann A, Geiran OR, Bugge JF: Renal function after cardiopulmonary bypass surgery in patients with impaired renal function. A randomized study of the effect of nifedipine. Eur J Anaesthesiol 2008; 25:319-325

83 Woo EB, Tang AT, el-Gamel A, et al: Dopamine therapy for patients at risk of renal dysfunction following cardiac surgery: science or fiction? Eur J Cardiothorac Surg 2002;22: 106-111.

84 Yallop KG, Sheppard SV, Smith DC: The effect of mannitol on renal function following cardio-pulmonary bypass in patients with normal pre-operative creatinine. Anaesthesia 2008;63:576-582.

85 Yavuz S, Ayabakan N, Dilek K, Ozdemir A: Renal dose dopamine in open heart surgery. Does it protect renal tubular function? J Cardiovasc Surg 2002;43:25-30.

86 Yavuz S, Ayabakan N, Goncu MT, Ozdemir IA: Effect of combined dopamine and diltiazem on renal function after cardiac surgery. Med Sci Monit 2002;8:P45-P50.

-87 Parikh CR, Garg AX: Acute kidney injury: Better biomarkers and beyond. Kidney Int 2008;73:801-803

-88 Furnary AP, Wu Y, Hiratzka LF, Grunkemeier GL, Page US 3rd: Aprotinin does not increase the risk of renal failure in cardiac surgery patients. Circulation 2007;116:127-133.

89 Mehta RL, Kellum JA, Shah SV, et al: Acute Kidney Injury Network: report of an initiative to improve acute kidney injury outcomes. Crit Care 2007;11:R31. 